\title{
Revealing the Underlying Mechanisms of Stacking Order and Interlayer Magnetism in Bilayer $\mathrm{CrBr}_{3}$
}

\author{
Jun-Shan Si, Hongxing Li, Bin-Guang He, Zi-Peng Cheng, and Wei-Bing Zhang* \\ Hunan Provincial Key Laboratory of Flexible Electronic Materials Genome Engineering, \\ School of Physics and Electronic Sciences, Changsha University of Science and Technology, \\ Changsha 410114, People's Republic of China. \\ E-mail: zhangwb@csust.edu.cn
}

\begin{abstract}
Aiming to clarify the mechanisms governing the interlayer magnetic coupling, we have investigated the stacking energy and interlayer magnetism of bilayer $\mathrm{CrBr}_{3}$ systemically. The magnetic ground states of bilayer $\mathrm{CrBr}_{3}$ with different R-type and H-type stacking orders are established, which is found to be in good agreement with recent experiment (Science 366,983(2019)).Further analyses indicate that the stacking energy is mainly determined by the Coulomb interaction between the interlayer nearest-neighbor $\mathrm{Br}-\mathrm{Br}$ atoms. While interlayer magnetism can be understood by a competition between super-super-exchange interactions involving $t_{2 g}-t_{2 g}$ and $t_{2 g}-e_{g}$ orbitals and semi-covalent exchange interactions of $e_{g}-e_{g}$ orbitals. Our studies give an insightful understanding for stacking order and interlayer magnetism of bilayer $\mathrm{CrBr}_{3}$, which should be useful to understand quantum confinement effect of other layered magnets in two-dimensional limit.
\end{abstract}




\section{Keywords}

stacking order, interlayer magnetism, $\mathrm{CrBr}_{3}$, super-superexchange, 2D magnet

The discovery of intrinsic ferromagnetism (FM) in atomically thin crystals has triggered increasing interest in two-dimensional (2D) magnetism. ${ }^{1-6}$ As the first Ising-type single-layer ferromagnetic semiconductor realized experimentally, ${ }^{1} \mathrm{CrI}_{3}$ has attracted special interest due to the novel quantum confinement effect. Bulk $\mathrm{CrI}_{3}$ shows ferromagnetic (FM) order both within and between layers, ${ }^{7-9}$ whereas its few-layer samples possess interlayer antiferromagnetism. ${ }^{10-12}$ This layer-dependent interlayer magnetic order leads to a number of emerging exotic phenomena ${ }^{13-19}$ including giant magnetoresistance in magnetic tunnel device and nonreciprocal second-order nonlinear optical effect. Although recent experimental ${ }^{10-12}$ and theoretical studies ${ }^{20-23}$ have confirmed a clear correlation between the magnetic ground state and the stacking order, the underlying mechanism is not yet understood. ${ }^{24}$

$\mathrm{CrBr}_{3}$ is another important vdW magnetic material, which has been known as the first ferromagnetic semiconductor. ${ }^{25}$ The rise of $2 \mathrm{D}$ magnetism also intrigues extensive research on the magnetism of $\mathrm{CrBr}_{3}$ in two-dimensional limit. ${ }^{24,26,27}$ The interlayer coupling in atomically thin $\mathrm{CrBr}_{3}$ is found to be ferromagnetic, ${ }^{24,26,27}$ which is distinct from $\mathrm{CrI}_{3}$. Significant difference between $\mathrm{CrBr}_{3}$ and $\mathrm{CrI}_{3}$ evokes the further understanding of interlayer magnetism. To reveal the underlying mechanism, Chen et al. ${ }^{24}$ have grown the bilayer $\mathrm{CrBr}_{3}$ with various stacking orders through molecular beam epitaxy methods, and observed stacking-dependent interlayer magnetism directly. This interesting experimental result provides an excellent platform to study and verify the magnetic mechanism in van der Waals bilayers.

Similar with $\mathrm{CrI}_{3}$, the bulk $\mathrm{CrBr}_{3}$ has a low-temperature phase with the space group $\mathrm{R} \overline{3}$ and a high-temperature phase with space group $C 2 / m \cdot{ }^{7,28}$ As shown in Fig. 1, Cr atoms

of monolayer $\mathrm{CrBr}_{3}$ are arranged in a honeycomb lattice and each atom is surrounded by an octahedron of six Br atoms. Within a single honeycomb formed by six Cr atoms, there are three top and bottom $\mathrm{Br}$ atoms marked by solid and dotted red triangles with opposite orientations, respectively. Due to the weak interlayer vdW interaction, different stacking 

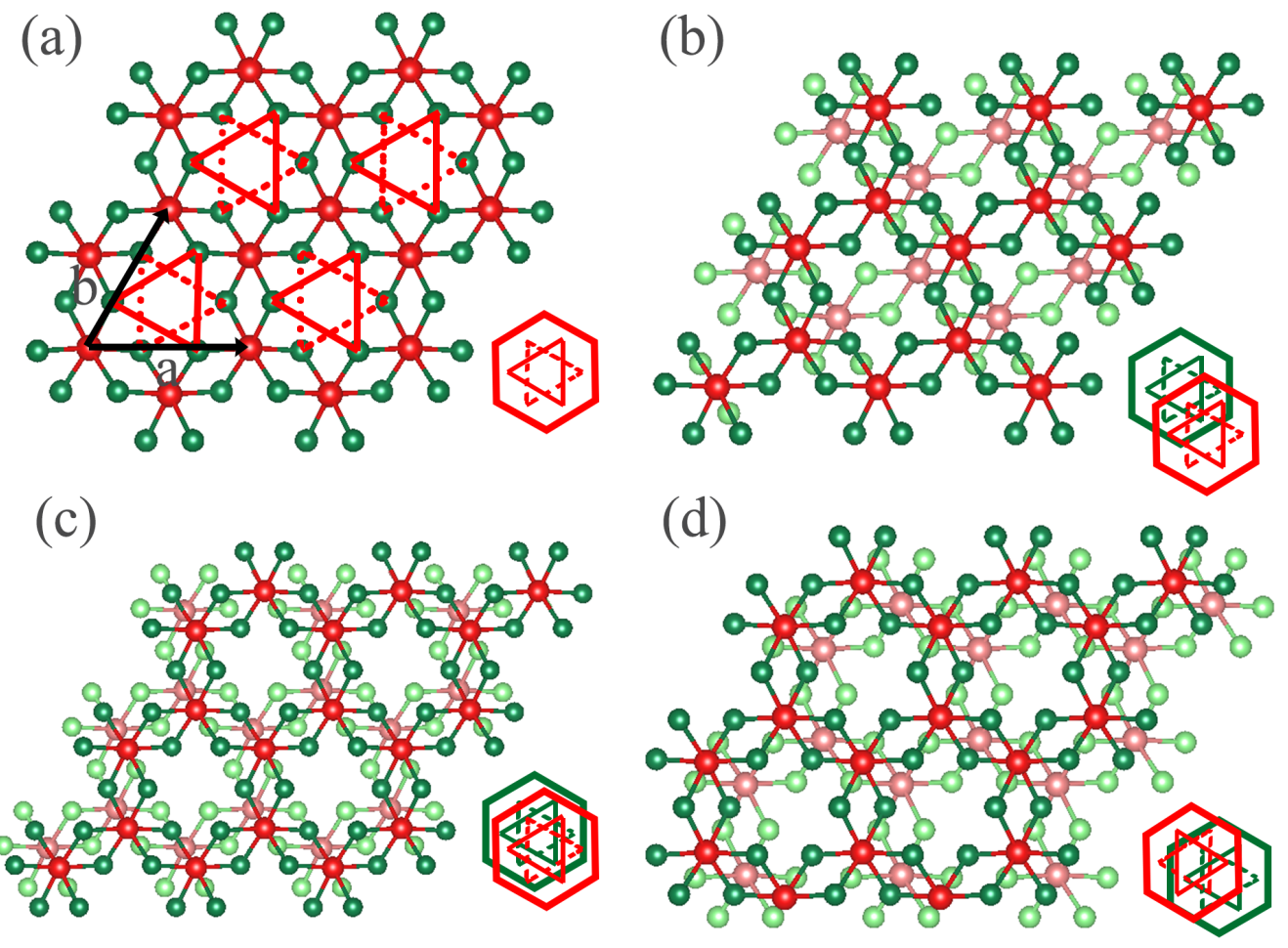

Figure 1: (Color online) The crystal structure of bilayer $\mathrm{CrBr}_{3}$ with different stacking orders. (a) The top view of monolayer $\mathrm{CrBr}_{3}$. Within the $\mathrm{Cr}$ honeycomb lattice, the top and bottom surfaces of Br atoms form single triangles but with opposite orientation, marked in solid and dotted red lines, respectively. The top view of three bilayer $\mathrm{CrBr}_{3}$ with different stacking $\mathrm{R}_{33}, \mathrm{R}_{15}, \mathrm{H}_{41}$ are shown in (b),(c) and (d) respectively. 
orders are easy to form. As labeled in recent experimental work, ${ }^{24}$ there are two kinds of stacking orders, namely R-type and H-type. Both layers align to the same orientation in the R-type but rotate by a $180^{\circ}$ in H-type stacking order. To obtain the details of stacking energy and interlayer exchange energy, a uniform $6 \times 6$ displacement vector grid in surface unit cell, which corresponds to 36 different stacking structures $\mathrm{R}_{m n}\left(\mathrm{H}_{m n}\right)$, was considered in the calculation. $\mathrm{R}_{m n}\left(\mathrm{H}_{m n}\right)$ means that the upper layer is shifted by $\frac{m}{6} \vec{a}+\frac{n}{6} \vec{b}$ relative to the AA-stacked R-type(H-type) bilayer, in which $\vec{a}$ and $\vec{b}$ are the surface unit vectors respectively. Three typical structures $\mathrm{R}_{33}, \mathrm{R}_{15}$ and $\mathrm{H}_{41}$, which are prepared in recent experiment successfully, are shown in Fig. 1-(b) to -(d) for clear illustration.

All DFT calculations are performed using the Vienna simulation package (VASP) code ${ }^{29,30}$ within the projector augmented-wave (PAW) method. ${ }^{31,32}$ General gradient approximations (GGA) both in PBEsol ${ }^{33}$ and PBE implementations ${ }^{34}$ are adopted as the exchange correlation functional. A plane-wave basis set with a cutoff energy of $700 \mathrm{eV}$ and a $\Gamma$-centered $17 \times 17 \times 1$ Monkhorst-Pack grid are used in the calculations. A simple rotationally invariant $\mathrm{DFT}+\mathrm{U}$ method $^{35}$ with effective on-site Coulomb interaction $U-J$ of $2.8 \mathrm{eV}(\mathrm{U}=3.9 \mathrm{eV}$, $\mathrm{J}=1.1 \mathrm{eV}$ ) is chosen for the $\mathrm{Cr}$ atoms to account for strong electronic correlations. ${ }^{20}$ Atomic positions are optimized with a force convergence tolerance of $3 \mathrm{meV} / \AA$. A vacuum spacing of $20 \AA$ is used in the supercell to avoid interaction between images. The phonon calculations have been performed using the finite-displacement method, as implemented in the Phonopy code, ${ }^{36}$ in which a $2 \times 2 \times 1$ supercell is used.

Starting from the fully relaxed $\mathrm{R}_{22}$ structure, we constructed bilayer $\mathrm{CrBr}_{3}$ with various stacking orders by rigidly shifting the upper atomic layer along $\vec{a}$ and $\vec{b}$ direction. The stacking energies, defined as the energy difference between different stacking and AA-stacking structures, are shown in Fig. 2. We can find that the change trend of stacking energy for R-type $\mathrm{CrBr}_{3}$ is similar to that of $\mathrm{CrI}_{3},{ }^{20}$ but the energy difference is much smaller. This implies that formation of stacking order in $\mathrm{CrBr}_{3}$ is much easier, which also consists with the diversity of interlayer stacking orders found in recent MBE experiment. ${ }^{24}$ 

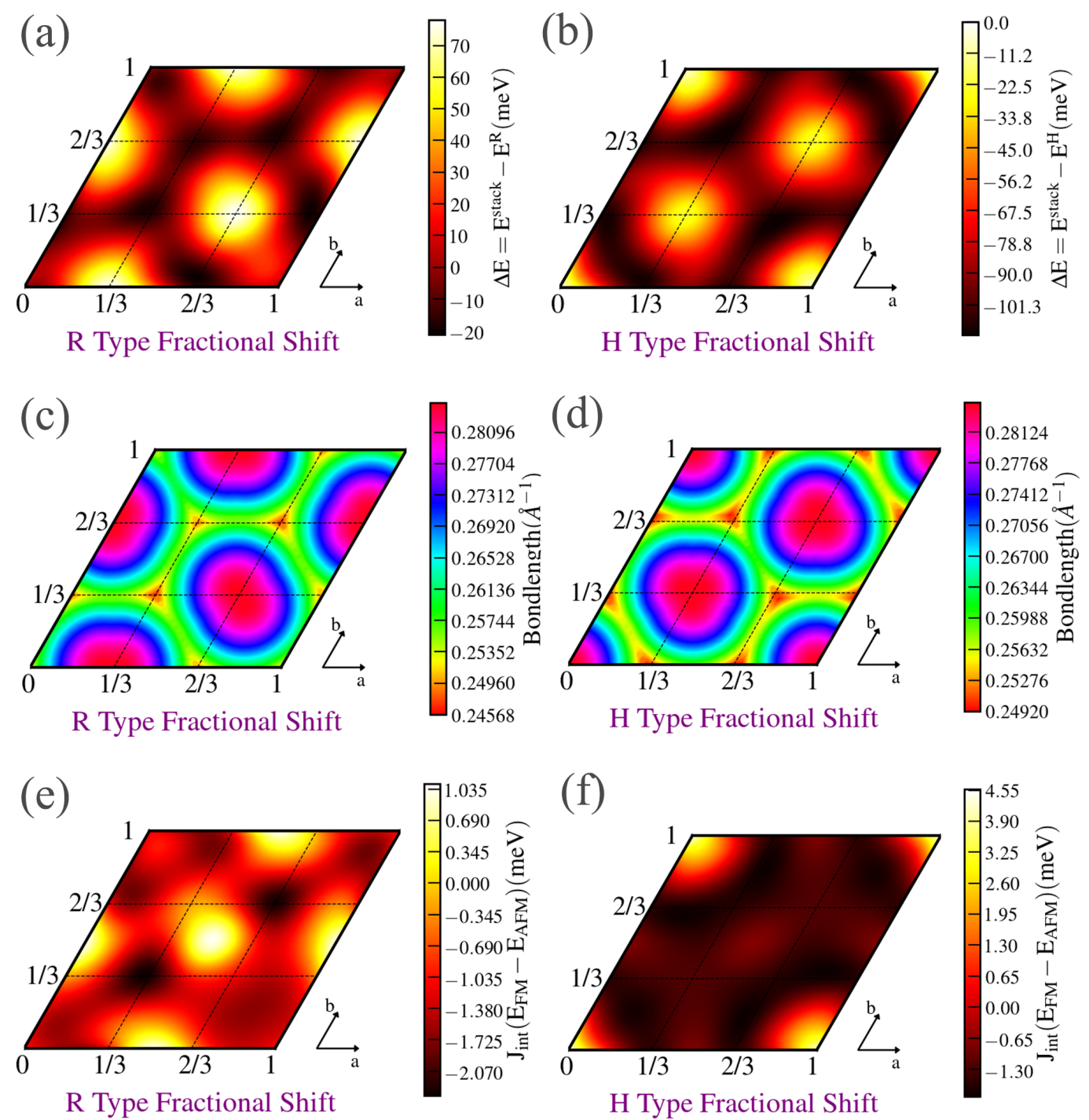

Figure 2: (Color online) The stacking energy, interlayer nearest-neighbor(NN) Br-Br distance and interlayer exchange energy as a function of lateral shift, with respect to AA-stacking R-type and H-type bilayer $\mathrm{CrBr}_{3}$. (a),(c) and (e) represent the results of R-type stacking configures, while the corresponding results for H-type are shown in (b),(d) and (f). The heatmaps were drawn by interpolating over the neighboring data points based on computational data obtained using a $6 \times 6$ grid. 
Moreover, we can also find that $\mathrm{R}_{22}$ and $\mathrm{R}_{24}$ locate at energy minimum of potential energy surface, which corresponds to the stacking order of $\mathrm{R} \overline{3}$ and $C 2 / m$ bulk phases, respectively. It should be noticed that three structures $\mathrm{R}_{33}, \mathrm{R}_{15}$ and $\mathrm{H}_{41}$ identified in recent experiments do not locate in the local minimum. However, the energy differences between various stacking orders are tiny, which implies a similar energetic stability. To further evaluate their stability, we also calculated the phonon spectrum of three kinds of structure. As shown in Fig. S1, there are essentially no imaginary frequencies in the whole Brillouin zone, which indicates that these stacking structures are dynamically stable and should be feasible in experiment.

To reveal the underlying mechanism governing stacking energy, we plot the variance of the reciprocal of the distance $\left(\frac{1}{r}\right)$ between two nearest-neighbor(NN) Br atoms in different single-layers. As shown in Fig. 2-(c) and (d), the variance of $\frac{1}{r}$ are highly similar to the stacking energy surfaces. Moreover, we can even judge the relative stacking energy using the distance of interlayer $\mathrm{NN} B r$ atoms. For example, a vary small barrier between $R_{22}$ and $R_{24}$ can be found in Fig. 2-(c), which agrees with the stacking energy surface (Fig. 2-(a)). Since the Coulomb potential of two atoms is known to be proportional to $\frac{1}{r}$, we thus conclude that the stacking energy is mainly contributed by the Coulomb interaction between the interlayer NN Br atoms.

We now turn our attention to the interlayer magnetic order of bilayer $\mathrm{CrBr}_{3}$ with different stacking orders. The interlayer exchange energies, defined as the energy difference between the interlayer ferromagnetic and anti-ferromagnetic spin configurations, are calculated and shown in Fig. 2-(e) and (f). Since interlayer exchange energy is very subtle, we also used different methods to validate our results. Firstly, we check the variance of interlayer exchange energy with Coulomb interaction $\mathrm{U}$ in $\mathrm{LDA}+\mathrm{U}$ methods. As shown in the Fig. S2, although the exchange energy change slightly but the interlayer magnetic order keeps invariant. Moreover, further calculations including the spin-orbital coupling (SOC) effect and using PBE functional have also been performed for comparison. As shown in Table. 1, all methods predict the consistent interlayer magnetic order, indicating the reliability 
of the present investigation. Our calculations indicate both $R_{22}$ and $R_{24}$ structures, which correspond to the stacking order of bulk $\mathrm{R} \overline{3}$ and $C 2 / m$ phases, show interlayer ferromagnetism. This agrees with recent tunneling measurements, ${ }^{26}$ in which the interlayer coupling in atomically thin $\mathrm{CrBr}_{3}$ is found to be ferromagnetic. Moreover, $\mathrm{R}_{33}$ and $\mathrm{H}_{41}$ stacking was found to be antiferromagnetic and ferromagnetic, which is also consistent with the recent experimental results. ${ }^{24}$ However, the experimentally identified anti-ferromagnetic $R_{15}$ phase was predicted to be interlayer ferromagnetic. Considered the agreement between experiment and theory about other stacking orders, further experimental verification about interlayer magnetism of $\mathrm{R}_{15}$-stacking is highly required.

Table 1

Interlayer exchange energy in unit of meV of bilayer $\mathrm{CrBr}_{3}$ with different stacking orders calculated using four methods.

\begin{tabular}{ccccc}
\hline Type & PBE $+\mathrm{U}$ & $\mathrm{PBE}+\mathrm{U}+\mathrm{SOC}$ & $\mathrm{PBEsol}+\mathrm{U}$ & $\mathrm{PBEsol}+\mathrm{U}+\mathrm{SOC}$ \\
\hline Hollow II $\left(\mathrm{R}_{22}\right)$ & -1.896 & -2.146 & -2.305 & -2.001 \\
Hollow III $\left(\mathrm{R}_{24}\right)$ & -0.189 & -0.192 & -0.261 & -0.209 \\
Special $\left(\mathrm{R}_{33}\right)$ & 0.942 & 0.832 & 1.077 & 1.006 \\
Bridge I $\left(\mathrm{R}_{15}\right)$ & -1.076 & -1.242 & -1.312 & -1.103 \\
Bridge I $\left(\mathrm{H}_{41}\right)$ & -1.476 & -1.651 & -1.760 & -1.643 \\
\hline
\end{tabular}

In addition, we also studied the influence of layer distance on interlayer magnetism. As shown in the fig. 3-(a) and -(b). We can find a interesting stacking-dependent behaviour. For example, the reduction of distance enhances interlayer ferromagnetism for $\mathrm{R}_{22}$ and $\mathrm{H}_{41}$, but enhances anti-ferromagnetism in case of $\mathrm{R}_{33}$ and $\mathrm{H}_{00}$ phase. Moreover, the change of layer distance will tune the interlayer magnetic state between FM and AFM of $\mathrm{H}_{22}$ and $\mathrm{R}_{20}$ stacking as shown in Fig. 3-(b). This change trend is similar to the potential energy curve of diatomic molecules, which indicates that there may be two kind of competition i.e. ferromagnetism and anti-ferromagnetism governing the interlayer magnetic coupling in bilayer $\mathrm{CrBr}_{3}$.

Now, let's focus on the microscopic mechanism of interlayer magnetic coupling. As established in the earlier study, the $\mathrm{Cr}^{3+}$ ions in $\mathrm{CrBr}_{3}$ exhibit a high-spin $\mathrm{t}_{2 g}^{3} \mathrm{e}_{g}^{0}$ electronic 

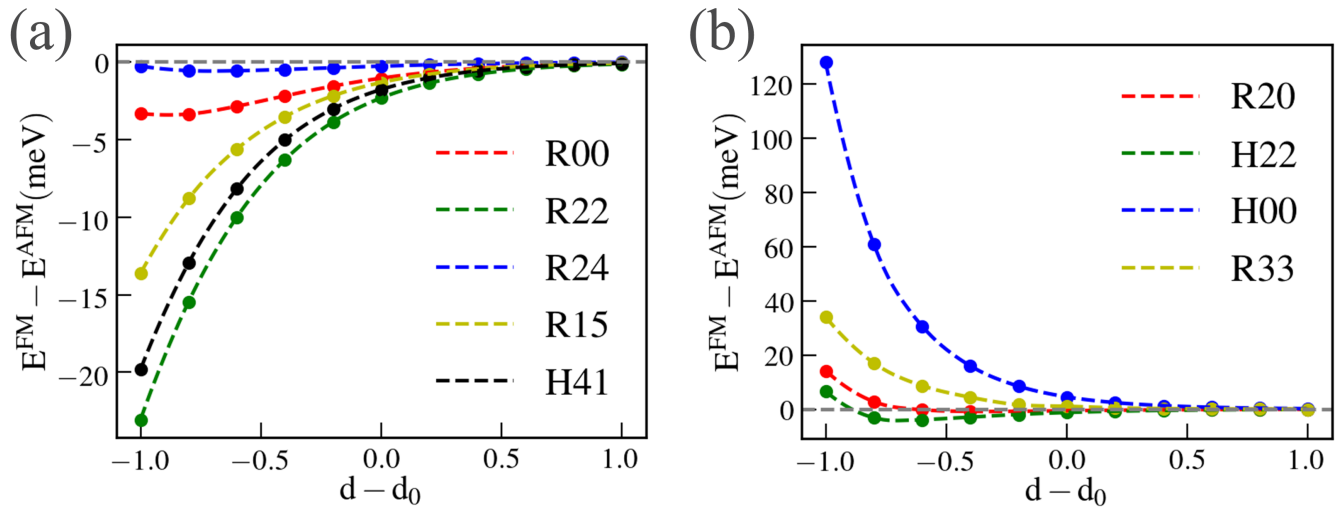

Figure 3: (Color online) The evolution of interlayer exchange energy with layer distances for different stacking orders.

configuration. ${ }^{9}$ The intra-layer magnetic coupling is predominated by the $\mathrm{Cr}-\mathrm{Br}-\mathrm{Cr}$ superexchange interaction. For the interlayer magnetic coupling, there are several possible exchange interactions. Since the distance between $\mathrm{Cr}$ atoms in different layers are much larger than common super-exchange path, the cations are separated by two anions in the possible super-exchange path, forming an $\mathrm{Cr}-\mathrm{Br}-\mathrm{Br}-\mathrm{Cr}$ interaction. The well-known GoodenoughKanamori-Anderson(GKA) rule should be qualified to understand this interaction. ${ }^{37}$ Fig. 4(a) shows a schematic diagram of different exchange interactions between interlayer $\mathrm{Cr}$ atoms. $\mathrm{t}_{2 g}-\mathrm{t}_{2 g}$ transitions are prohibited for ferromagnetic coupling but allowed for antiferromagnetic coupling. On the other hand, due to the local Hund rule, the hopping of $\mathrm{t}_{2 g}-\mathrm{e}_{g}$ leads to the ferromagnetic exchange coupling. In addition, although the two empty $\mathrm{e}_{g}-\mathrm{e}_{g}$ orbitals can't give super-exchange-like interaction, they can give anti-ferromagnetic $\sigma$ bond spin-spin interactions via a purely semi-covalent-exchange interaction. ${ }^{37}$ Recent orbitalresolved magnetic exchange interaction calculations of $\mathrm{CrI}_{3}{ }^{22}$ also give the similar pictures, in which $\mathrm{t}_{2 g}-\mathrm{t}_{2 g}, \mathrm{t}_{2 g}-\mathrm{e}_{g}$, and $\mathrm{e}_{g}-\mathrm{e}_{g}$ interactions are found to be anti-ferromagnetic, ferromagnetic and anti-ferromagnetic, respectively.

Clearly, the interlayer magnetic coupling of $\mathrm{CrBr}_{3}$ can be understood by the competition of these three interactions. According to the symmetry matching principle of molecular orbital, $\mathrm{e}_{g}$ and $\mathrm{t}_{2 g}$ orbital of Cr-3d electron can hybridize with $\sigma$ and $\pi$ state of the ligands 


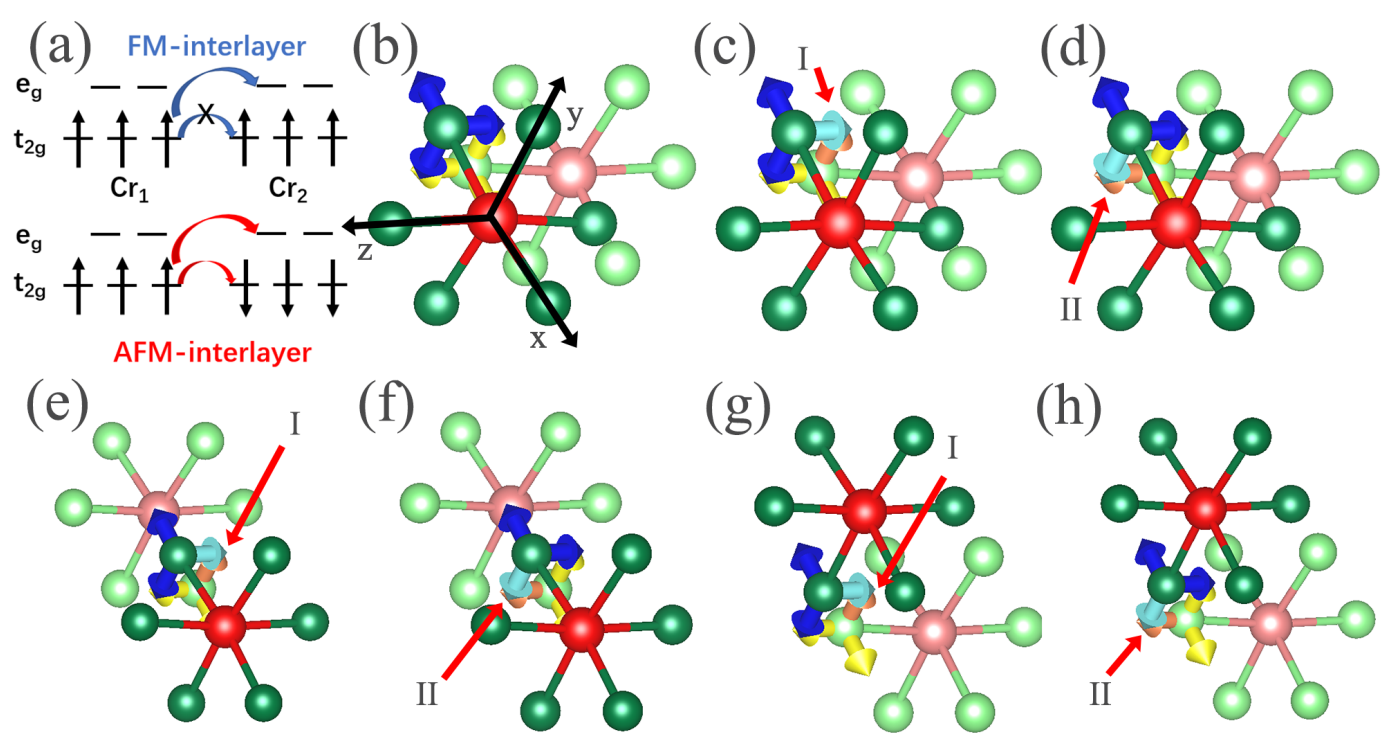

Figure 4: (Color online) The interlayer magnetic exchange mechanism. (a) A schematic diagram of the orbital dependent interlayer super-super-exchange interactions. Hopping of the form $t_{2 g}-t_{2 g}$ is prohibited in FM exchange (blue) but allowed in AFM exchange (red). Three directions of $\mathrm{p}$ orbital of the nearest-neighbors $\mathrm{Br}$ atoms are shown in (b). The nearest-neighbors interlayer interactions are shown in (c) and (d). (e)-(h) represent the next-nearest-neighbors interlayer interaction.

$\mathrm{Br}$ atoms, respectively. Since the $\sigma$ bond is known to be stronger than $\pi$ bond, the strength of $\mathrm{e}_{g}-\mathrm{e}_{g}$ interaction is expected to be greater than that of the $\mathrm{t}_{2 g}-\mathrm{e}_{g}$, while the $\mathrm{t}_{2 g}-\mathrm{t}_{2 g}$ involves two p- $\pi$ orbitals should be weakest under the same conditions. This conclusion is also supported by recent research about interlayer magnetism of $\mathrm{CrI}_{3} \cdot{ }^{22}$

Due to the intermediation role of $\mathrm{Br}-\mathrm{Br}$ atoms, whether two p orbitals of interlayer NN $\mathrm{Br}$ atoms interact with each other is curial to form the possible exchange interaction. As shown above, the local coordinate system of octahedral environment is different from the global cartesian coordinate. The coordinate system of the two monolayers should be identical for R-type stacking but different for H-type stacking order, as shown in Fig. S3. When the directions of two p orbitals intersect, the wavefunction of two p orbitals should be overlapped and possible hopping can happen (Fig. S3-(b) and (c). However, if the directions of two $\mathrm{p}$ orbitals do not intersect, no interaction would occur due to their large distance.(Fig. S3-(a) and (d)). 
Taking the $\mathrm{R}_{33}$ structure as an example, we illustrate the above idea to understand the interlayer magnetic coupling of bilayer $\mathrm{CrBr}_{3}$. As shown in Fig. 4-(b), there are three different directions for p- orbital of each $\mathrm{Br}$ atoms for nearest-neighbor(NN) Cr-Br-Br-Cr path, in which the blue and yellow arrows represent the p-orbital in upper and lower atomic layer, respectively. According to the above assumption, two groups of orbitals labeled by I (Fig. 4(c)) and II (Fig. 4-(d)) are expected to interact with each other. And the corresponding $\mathrm{d}$ orbital, which hybridizes with the above p orbital, can be determined according to the principle of symmetry matching. For group I, both the p orbital of the upper and lower $\mathrm{Br}$ atom are perpendicular to the Br-I bond. These $\mathrm{p}-\pi$-like molecular orbital will hybrid with the $\mathrm{t}_{2 g}$ orbital of $\mathrm{Cr}$ atom, which forms a $\mathrm{t}_{2 g} \mathrm{t}_{2 g}$ interaction. For group II, the p- $\pi$-like orbital of the upper $\mathrm{Br}$ atom can only bind with the $\mathrm{t}_{2 g}$ orbital, while the $\mathrm{p}-\sigma$-like orbital of the lower $\mathrm{Br}$ atom would form molecular orbital with the $\mathrm{e}_{g}$ orbital of $\mathrm{Cr}$ atom. The interaction between the next-nearest-neighbors (NNN) $\mathrm{Cr}$ atoms is similar to that of NN. As shown in the Fig. 4-(e) to Fig. 4-(h), there are two non-equivalent $\mathrm{Cr}-\mathrm{Br}-\mathrm{Br}-\mathrm{Cr}$ channels between the NNN Cr atoms. For channel 1 (Fig. 4-(e) and -(f)), according to the previous analysis, it can be obtained that both groups I and II have $t_{2 g}$ - $t_{2 g}$ interaction. For channel 2, group I and group II have $\mathrm{t}_{2 g}-\mathrm{t}_{2 g}$ and $\mathrm{e}_{g}-\mathrm{e}_{g}$ interaction, respectively. Totally, there were 6 $\mathrm{t}_{2 g}-\mathrm{t}_{2 g}$ and $6 \mathrm{t}_{2 g}-\mathrm{e}_{g}$ interactions between the NN Cr atoms in a single cell with $\mathrm{R}_{33}$ structure containing $4 \mathrm{Cr}$ atoms. And $14 \mathrm{t}_{2 g}-\mathrm{t}_{2 g}$ and $6 \mathrm{e}_{g}-\mathrm{e}_{g}$ interactions are present between the NNN Cr atoms. Since the $\mathrm{t}_{2 g}-\mathrm{t}_{2 g}$ interaction is much weaker, $\mathrm{t}_{2 g}-\mathrm{e}_{g}$ and $\mathrm{e}_{g}-\mathrm{e}_{g}$ will be determinative. Considered the $\mathrm{e}_{g}-\mathrm{e}_{g}$ is stronger than $\mathrm{t}_{2 g}-\mathrm{e}_{g}$ interaction and the number of both interactions are identical, the interlayer magnetic coupling of $R_{33}$ should be anti-ferromagnetic.

In Table. 2, we list the number of these three interactions in other stacking structures. For $\mathrm{R}_{22}$-stacking, there are $6 \mathrm{NN} \mathrm{t}_{2 g}-\mathrm{t}_{2 g}, 15 \mathrm{NNN} \mathrm{t}_{2 g}$ - $\mathrm{t}_{2 g}$ and $30 \mathrm{NNN} \mathrm{t}_{2 g}-\mathrm{e}_{g}$ super-super-exchange interactions. Considered the $\mathrm{t}_{2 g}-\mathrm{e}_{g}$ interaction is stronger than $\mathrm{t}_{2 g}-\mathrm{t}_{2 g}$, the $30 \mathrm{NNN} t_{2 g}-\mathrm{e}_{g}$ interaction should be determinative and the interlayer magnetic coupling of $R_{22}$ should be ferromagnetic. In the $\mathrm{R}_{24}$ structure, $12 \mathrm{NN} \mathrm{t}_{2 g} \mathrm{t}_{2 g}, 24 \mathrm{NN} \mathrm{t}_{2 g^{-}} \mathrm{e}_{g}, 7 \mathrm{NNN} \mathrm{t}_{2 g^{-}} \mathrm{t}_{2 g}$ and $7 \mathrm{NNN} \mathrm{e}_{g^{-}}$ 
Table 2

The interlayer NN and NNN exchange interactions between $\mathrm{Cr}$ atoms and the corresponding number for different stacking orders.

\begin{tabular}{cccccccc}
\hline Stacking & States & Path & Number & Type & Number & Type & Number \\
\hline $\mathrm{R}_{22}$ & FM & NN & 1 & $\mathrm{t}_{2 g}-\mathrm{t}_{2 g}$ & 6 & & \\
& & NNN & 15 & $\mathrm{t}_{2 g}-\mathrm{t}_{2 g}$ & 15 & $\mathrm{t}_{2 g}-\mathrm{e}_{g}$ & 30 \\
$\mathrm{R}_{24}$ & FM & NN & 6 & $\mathrm{t}_{2 g}-\mathrm{t}_{2 g}$ & 12 & $\mathrm{t}_{2 g}-\mathrm{e}_{g}$ & 24 \\
& & $\mathrm{NNN}$ & 7 & $\mathrm{t}_{2 g}-\mathrm{t}_{2 g}$ & 7 & $\mathrm{e}_{g}-\mathrm{e}_{g}$ & 7 \\
$\mathrm{R}_{33}$ & \multirow{2}{*}{$\mathrm{AFM}$} & $\mathrm{NN}$ & 3 & $\mathrm{t}_{2 g}-\mathrm{t}_{2 g}$ & 6 & $\mathrm{t}_{2 g}-\mathrm{e}_{g}$ & 6 \\
& & $\mathrm{NNN}$ & 7 & $\mathrm{t}_{2 g}-\mathrm{t}_{2 g}$ & 14 & $\mathrm{e}_{g}-\mathrm{e}_{g}$ & 6 \\
$\mathrm{H}_{41}$ & \multirow{2}{*}{$\mathrm{FM}$} & $\mathrm{NN}$ & 4 & $\mathrm{t}_{2 g}-\mathrm{t}_{2 g}$ & 8 & $\mathrm{t}_{2 g}-\mathrm{e}_{g}$ & 8 \\
& & $\mathrm{NNN}$ & 3 & $\mathrm{t}_{2 g}-\mathrm{t}_{2 g}$ & 12 & & \\
$\mathrm{H}_{00}$ & \multirow{2}{*}{$\mathrm{AFM}$} & $\mathrm{NN}$ & 2 & $\mathrm{t}_{2 g}-\mathrm{t}_{2 g}$ & 6 & $\mathrm{e}_{g}-\mathrm{e}_{g}$ & 6 \\
& & $\mathrm{NNN}$ & 6 & $\mathrm{t}_{2 g}-\mathrm{t}_{2 g}$ & 20 & $\mathrm{t}_{2 g}-\mathrm{e}_{g}$ & 10 \\
$\mathrm{H}_{22}$ & \multirow{2}{*}{$\mathrm{FM}$} & $\mathrm{NN}$ & 1 & $\mathrm{t}_{2 g}-\mathrm{t}_{2 g}$ & 3 & $\mathrm{e}_{g}-\mathrm{e}_{g}$ & 3 \\
& & $\mathrm{NNN}$ & 3 & $\mathrm{t}_{2 g}-\mathrm{t}_{2 g}$ & 10 & $\mathrm{t}_{2 g}-\mathrm{e}_{g}$ & 20 \\
\hline
\end{tabular}

$\mathrm{e}_{g}$ interactions are present. So the interlayer magnetic coupling of $\mathrm{R}_{24}$ stacking is decided by the competition between $\mathrm{e}_{g}-\mathrm{e}_{g}$ and $\mathrm{t}_{2 g}-\mathrm{e}_{g}$. Considered the larger number of $\mathrm{t}_{2 g}-\mathrm{e}_{g}$ interaction but the stronger interaction of $\mathrm{e}_{g}-\mathrm{e}_{g}$, the $\mathrm{R}_{24}$ structure is likely to be weakly ferromagnetic or anti-ferromagnetic, which are consistent with weak ferromagnetism predicted in our work. Recent experiment ${ }^{10-12}$ and theory ${ }^{20-23}$ indicate the interlayer magnetism of $\mathrm{CrI}_{3}$ in $\mathrm{R}_{24}$ $\operatorname{order}(C \mathcal{2} / m)$ is anti-ferromagnetic, which is also consistent with our analysis.

Moreover, the interlayer-distance-dependent magnetic properties of different stacking orders can be also understood by above mechanism. When interlayer distance reduced, interaction between $\mathrm{p}$ orbitals of $\mathrm{Br}$ atom will become stronger. For example, the interlayer magnetism of $\mathrm{R}_{22^{-}}$and H41-stacking are mainly decided by $\mathrm{t}_{2 g^{-}} \mathrm{e}_{g}$ due to the weak $\mathrm{t}_{2 g^{-}} \mathrm{t}_{2 g}$ interaction. The reduced distance will lead the interlayer exchange energy increase monotonously and ferromagnetism will be strengthened largely. For other stacking structure, in which two comparable exchange mechanisms present, changing layer distance may tune their interlayer magnetic state. The final magnetic order would be determined by the competing between two ferromagnetic and anti-ferromagnetic interactions, like in $\mathrm{R}_{20^{-}}$and $\mathrm{H}_{22}$-stacking.

It should be pointed out that microscopic mechanisms of interlayer magnetism in bi- 
layer $\mathrm{CrI}_{3}$ have been investigated by several groups. ${ }^{20-23}$ However, all these investigations mainly focused on the $\mathrm{R}_{22}$ and $\mathrm{R}_{24}$ orders of bilayer $\mathrm{CrI}_{3}$ while the interlayer magnetism of other stacking orders including H-type stacking is largely unexplored. In addition, most of the proposed mechanisms ${ }^{20,21}$ ignore the $\mathrm{e}_{g}-\mathrm{e}_{g}$ interaction, which is important for interlayer magnetism. The present study suggests a competing mechanism based on the principle of symmetry matching and the local orbital direction of $\mathrm{Br}$ atoms, which involves not only the $\mathrm{t}_{2 g}-\mathrm{t}_{2 g}$ and $\mathrm{t}_{2 g}-\mathrm{e}_{g}$ interactions but also $\mathrm{e}_{g}-\mathrm{e}_{g}$ interaction. Furthermore, our results can also give a consistent understanding for interlayer magnetism of bilayer $\mathrm{CrBr}_{3}$ with various stacking orders and other vdW bilayer magnets including $\mathrm{CrI}_{3}$.

In summary, we have revealed the underlying mechanism of the stacking order and interlayer magnetism of $\mathrm{CrBr}_{3}$ bilayer. Our results indicate that the stacking energy is closely correlated to the Coulomb potential between the interlayer $\mathrm{NN} \mathrm{Br}-\mathrm{Br}$ atoms. And the stacking-dependent interlayer magnetism can be understood by the competition of supersuper-exchange and semi-covalent exchange. The present work provides an insightful explanation for interlayer magnetism of $\mathrm{CrBr}_{3}$, which will be useful to understand the other layered magnetic semiconductors.

\section{Acknowledgement}

This work was supported by National Natural Science Foundation of China (Grant No.11874092 and No.11847157) the Fok Ying-Tong Education Foundation, China (Grant No. 161005), the Planned Science and Technology Project of Hunan Province (Grant No. 2017RS3034), Hunan Provincial Natural Science Foundation of China (Grant No. 2016JJ2001 and 2019JJ50636), and Scientific Research Fund of Hunan Provincial Education Department (Grant No. 18C0227). 


\section{References}

(1) Huang, B.; Clark, G.; Navarro-Moratalla, E.; Klein, D. R.; Cheng, R.; Seyler, K. L.; Zhong, D.; Schmidgall, E.; McGuire, M. A.; Cobden, D. H.; Yao, W.; Xiao, D.; JarilloHerrero, P.; Xu, X. Layer-dependent ferromagnetism in a van der Waals crystal down to the monolayer limit. Nature 2017, 546, 270-273.

(2) Gong, C.; Li, L.; Li, Z.; Ji, H.; Stern, A.; Xia, Y.; Cao, T.; Bao, W.; Wang, C.; Wang, Y.; Qiu, Z. Q.; Cava, R. J.; Louie, S. G.; Xia, J.; Zhang, X. Discovery of intrinsic ferromagnetism in two-dimensional van der Waals crystals. Nature 2017, 546, 265-269.

(3) Burch, K. S.; Mandrus, D.; Park, J.-G. Magnetism in two-dimensional van der Waals materials. Nature 2018, 563, 47-52.

(4) Gibertini, M.; Koperski, M.; Morpurgo, A. F.; Novoselov, K. S. Magnetic 2D materials and heterostructures. Nat. Nanotechnol. 2019, 14, 408-419.

(5) Gong, C.; Zhang, X. Two-dimensional magnetic crystals and emergent heterostructure devices. Science 2019, 363, eaav4450.

(6) Huang, B.; McGuire, M. A.; May, A. F.; Xiao, D.; Jarillo-Herrero, P.; Xu, X. Emergent phenomena and proximity effects in two-dimensional magnets and heterostructures. Nat. Mater. 2020, XXX, XXX (In press).

(7) McGuire, M. A.; Dixit, H.; Cooper, V. R.; Sales, B. C. Correction to Coupling of Crystal Structure and Magnetism in the Layered, Ferromagnetic Insulator CrI3. Chem. Mater. 2015, 27, 4165-4165.

(8) McGuire, M. A. Crystal and Magnetic Structures in Layered, Transition Metal Dihalides and Trihalides. Crystals 2017, \%, 121. 
(9) Zhang, W.-B.; Qu, Q.; Zhu, P.; Lam, C.-H. Robust intrinsic ferromagnetism and half semiconductivity in stable two-dimensional single-layer chromium trihalides. J. Mater. Chem. C 2015, 3, 12457-12468.

(10) Li, T.; Jiang, S.; Sivadas, N.; Wang, Z.; Xu, Y.; Weber, D.; Goldberger, J. E.; Watanabe, K.; Taniguchi, T.; Fennie, C. J.; Fai Mak, K.; Shan, J. Pressure-controlled interlayer magnetism in atomically thin CrI3. Nat. Mater. 2019, 18, 1303-1308.

(11) Thiel, L.; Wang, Z.; Tschudin, M. A.; Rohner, D.; Gutierrez-Lezama, I.; Ubrig, N.; Gibertini, M.; Giannini, E.; Morpurgo, A. F.; Maletinsky, P. Probing magnetism in 2D materials at the nanoscale with single-spin microscopy. Science 2019, 364, 973-+.

(12) Song, T. et al. Switching 2D magnetic states via pressure tuning of layer stacking. Nat. Mater. 2019, 18, 1298-1302.

(13) Klein, D. R.; MacNeill, D.; Lado, J. L.; Soriano, D.; Navarro-Moratalla, E.; Watanabe, K.; Taniguchi, T.; Manni, S.; Canfield, P.; Fernández-Rossier, J.; Jarillo-Herrero, P. Probing magnetism in 2D van der Waals crystalline insulators via electron tunneling. Science 2018, 360, 1218-1222.

(14) Song, T.; Cai, X.; Tu, M. W.-Y.; Zhang, X.; Huang, B.; Wilson, N. P.; Seyler, K. L.; Zhu, L.; Taniguchi, T.; Watanabe, K.; McGuire, M. A.; Cobden, D. H.; Xiao, D.; Yao, W.; Xu, X. Giant tunneling magnetoresistance in spin-filter van der Waals heterostructures. Science 2018, 360, 1214-1218.

(15) Wang, Z.; Gutiérrez-Lezama, I.; Ubrig, N.; Kroner, M.; Gibertini, M.; Taniguchi, T.; Watanabe, K.; Imamoğlu, A.; Giannini, E.; Morpurgo, A. F. Very large tunneling magnetoresistance in layered magnetic semiconductor CrI3. Nat. Commun. 2018, 9, 2516.

(16) Jiang, S.; Shan, J.; Mak, K. F. Electric-field switching of two-dimensional van der Waals magnets. Nat. Mater. 2018, 17, 406-410. 
(17) Huang, B.; Clark, G.; Klein, D. R.; MacNeill, D.; Navarro-Moratalla, E.; Seyler, K. L.; Wilson, N.; McGuire, M. A.; Cobden, D. H.; Xiao, D.; Yao, W.; Jarillo-Herrero, P.; $\mathrm{Xu}, \mathrm{X}$. Electrical control of 2D magnetism in bilayer CrI3. Nat. Nanotechno. 2018, 13, $544-548$.

(18) Jiang, S.; Li, L.; Wang, Z.; Mak, K. F.; Shan, J. Controlling magnetism in 2D CrI3 by electrostatic doping. Nat. Nanotechno. 2018, 13, 549-553.

(19) Sun, Z. et al. Giant nonreciprocal second-harmonic generation from antiferromagnetic bilayer CrI3. Nature 2019, 572, 497-501.

(20) Sivadas, N.; Okamoto, S.; Xu, X.; Fennie, C. J.; Xiao, D. Stacking-Dependent Magnetism in Bilayer CrI3. Nano Lett. 2018, 18, 7658-7664.

(21) Jiang, P.; Wang, C.; Chen, D.; Zhong, Z.; Yuan, Z.; Lu, Z.-Y.; Ji, W. Stacking tunable interlayer magnetism in bilayer $\mathrm{CrI}_{3}$. Phys. Rev. B 2019, 99, 144401.

(22) Jang, S. W.; Jeong, M. Y.; Yoon, H.; Ryee, S.; Han, M. J. Microscopic understanding of magnetic interactions in bilayer $\mathrm{CrI}_{3}$. Phys. Rev. Mater. 2019, 3, 031001.

(23) Soriano, D.; Cardoso, C.; Fernández-Rossier, J. Interplay between interlayer exchange and stacking in CrI3 bilayers. Solid State Commun. 2019, 299, 113662.

(24) Chen, W.; Sun, Z.; Wang, Z.; Gu, L.; Xu, X.; Wu, S.; Gao, C. Direct observation of van der Waals stacking-dependent interlayer magnetism. Science 2019, 366, 983-987.

(25) Tsubokawa, I. On the Magnetic Properties of a CrBr3 Single Crystal. J. Phys. Soc. Japan 1960, 15, 1664-1668.

(26) Ghazaryan, D. et al. Magnon-assisted tunnelling in van der Waals heterostructures based on CrBr3. Nat. Electron. 2018, 1, 344-349.

(27) Kim, H. H. et al. Evolution of interlayer and intralayer magnetism in three atomically thin chromium trihalides. P. Natl Acad. Sci. USA 2019, 116, 11131-11136. 
(28) Pollini, I. Electronic structure of $\mathrm{CrBr}_{3}$ studied by x-ray photoelectron spectroscopy. Phys. Rev. B 1999, 60, 16170-16175.

(29) Kresse, G.; Furthmüller, J. Efficiency of ab-initio total energy calculations for metals and semiconductors using a plane-wave basis set. Comp. Mater. Sci. 1996, 6, 15-50.

(30) Kresse, G.; Furthmüller, J. Efficient iterative schemes for ab initio total-energy calculations using a plane-wave basis set. Phys. Rev. B 1996, 54, 11169-11186.

(31) Blöchl, P. E. Projector augmented-wave method. Phys. Rev. B 1994, 50, 17953.

(32) Kresse, G.; Joubert, D. From ultrasoft pseudopotentials to the projector augmentedwave method. Phys. Rev. B 1999, 59, 1758-1775.

(33) Perdew, J. P.; Ruzsinszky, A.; Csonka, G. I.; Vydrov, O. A.; Scuseria, G. E.; Constantin, L. A.; Zhou, X.; Burke, K. Restoring the Density-Gradient Expansion for Exchange in Solids and Surfaces. Phys. Rev. Lett. 2008, 100, 136406.

(34) Perdew, J. P.; Burke, K.; Ernzerhof, M. Generalized Gradient Approximation Made Simple. Phys. Rev. Lett. 1996, 77, 3865-3868.

(35) Dudarev, S. L.; Botton, G. A.; Savrasov, S. Y.; Humphreys, C. J.; Sutton, A. P. Electron-energy-loss spectra and the structural stability of nickel oxide: An LSDA $+U$ study. Phys. Rev. B 1998, 57, 1505-1509.

(36) Togo, A.; Oba, F.; Tanaka, I. First-principles calculations of the ferroelastic transition between rutile-type and $\mathrm{CaCl} 2$-type $\mathrm{SiO} 2$ at high pressures. Phys. Rev. B 2008, 78, 134106.

(37) Goodenough, J. B. Magnetism and the Chemical Bond; Interscience Publishers: New York, 1963. 


\section{Supporting Information Available}

The following files are available free of charge.

- Fig. S1: Phonon dispersions of bilayer $\mathrm{CrBr}_{3}$ with $\mathrm{R}_{33}, \mathrm{R}_{15}$ and $\mathrm{H}_{41}$ stacking orders.

- Fig. S2: The evolution of interlayer exchange energy with Coulomb interaction parameter $\mathrm{U}$ in $\mathrm{LDA}+\mathrm{U}$ methods.

- Fig. S3: The sketch of p orbitals of interlayer NN Br atoms.
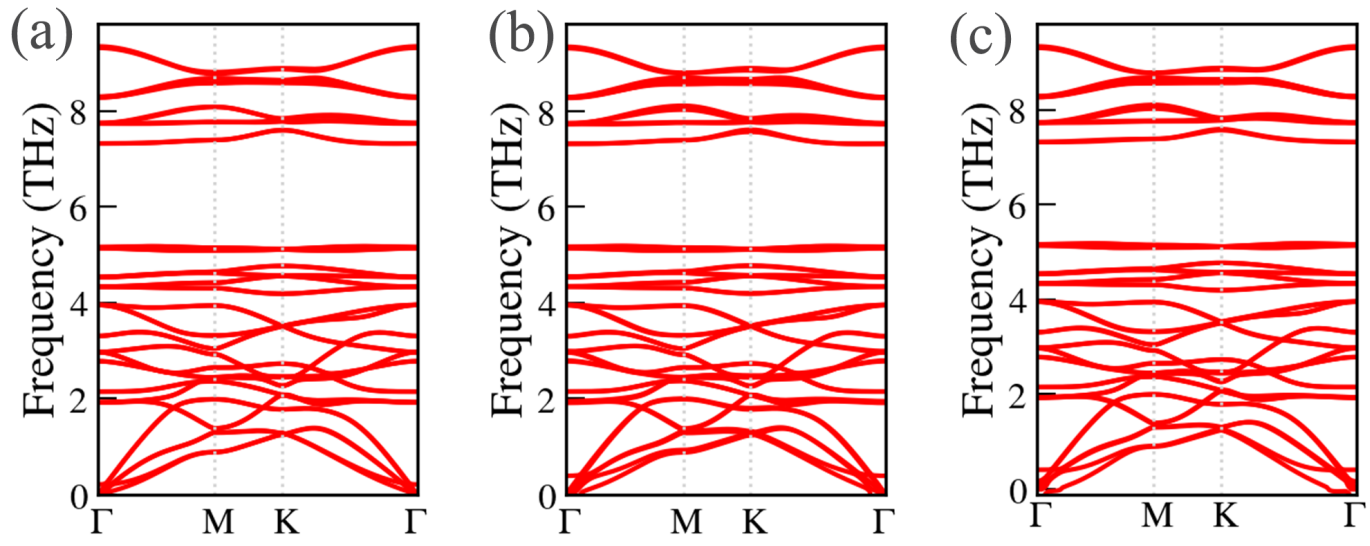

Figure S1: (Color online) Phonon dispersions of bilayer $\mathrm{CrBr}_{3}$ with $\mathrm{R}_{33}, \mathrm{R}_{15}$ and $\mathrm{H}_{41}$ stacking orders calculated using the PBE functional. 


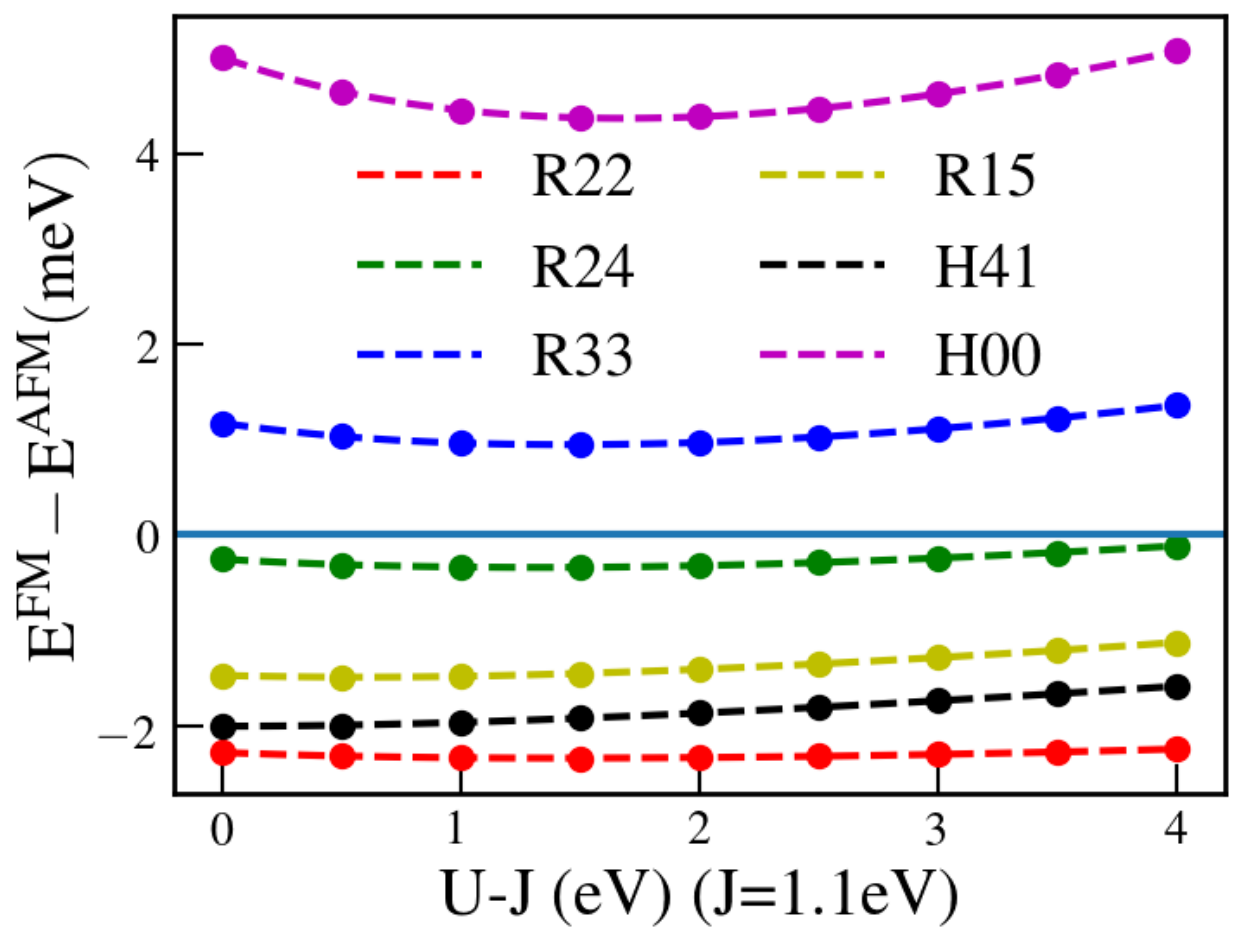

Figure S2: (Color online) The evolution of interlayer exchange energy with Coulomb interaction parameter $\mathrm{U}$ for different stacking configurations.

(a)

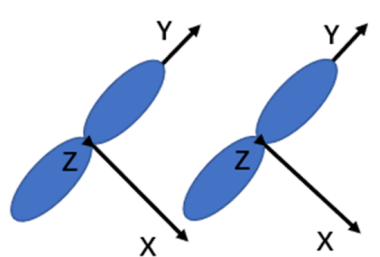

(c)

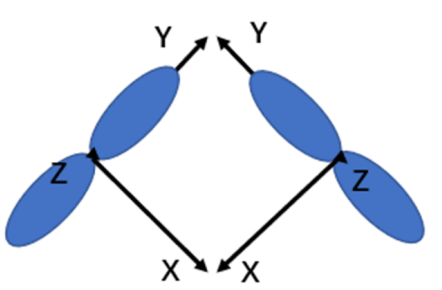

(b)

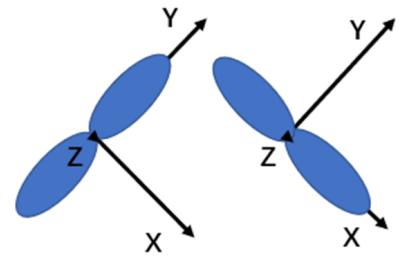

(d)

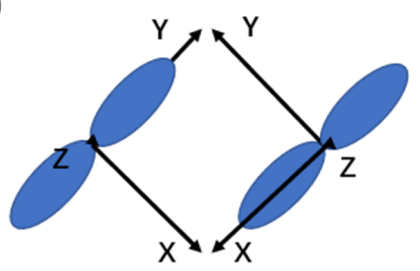

Figure S3: (Color online) The sketch of $\mathrm{p}$ orbitals of interlayer $\mathrm{NN} \mathrm{Br}$ atoms. (a) and (b) represent the relative directions of Br atoms in R-type stacking, while the (c) and (d) correspond to that in the H-type stacking. 


\section{Graphical TOC Entry}

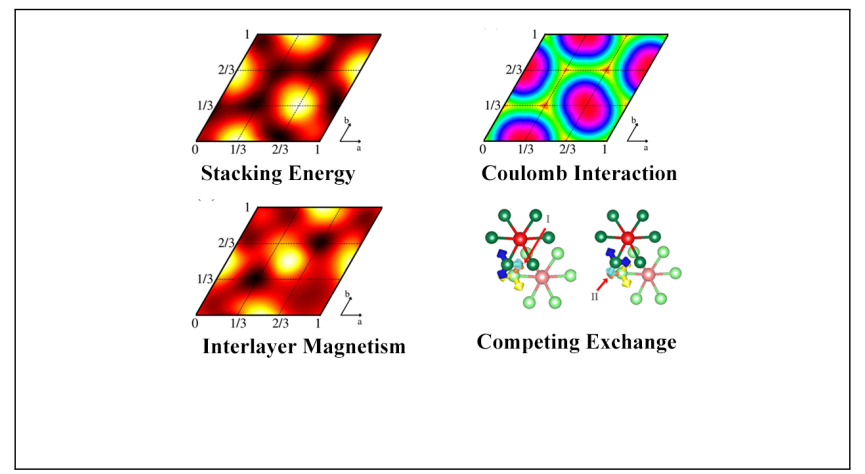

